Nouvelles perspectives en sciences sociales

\title{
Résilience d'un territoire face au chômage : les réseaux d'entreprises innovantes sur Montpellier
}

\section{Nicolas Bonnet}

Volume 5, numéro 1, octobre 2009

Sur le thème de la relation

URI : https://id.erudit.org/iderudit/038625ar

DOI : https://doi.org/10.7202/038625ar

Aller au sommaire du numéro

Éditeur(s)

Prise de parole

ISSN

1712-8307 (imprimé)

1918-7475 (numérique)

Découvrir la revue

Citer cet article

Bonnet, N. (2009). Résilience d'un territoire face au chômage : les réseaux d'entreprises innovantes sur Montpellier. Nouvelles perspectives en sciences sociales, 5(1), 97-115. https://doi.org/10.7202/038625ar
Résumé de l'article

L'objectif de l'article est d'évaluer la résilience d'un territoire face à des perturbations économiques, ici l'aire urbaine de Montpellier, au travers de l'étude de la morphologie des réseaux d'entreprises locales. Pour réaliser cette analyse, nous avons mené un recensement des dépôts communs de brevets des entreprises innovantes locales. Cette approche a permis une modélisation de ces réseaux avec la théorie des graphes sur plusieurs plages temporelles. Au final, cette analyse permet de mettre en évidence au sein de ces réseaux locaux d'innovation le rôle joué par des entreprises-pivots dans la résilience d'un territoire, notamment en termes de pérennité de son tissu entrepreneurial innovant mais également de niveau d'emploi. 


\title{
Résilience d'un territoire face au chômage : les réseaux d'entreprises innovantes sur Montpellier
}

\author{
Nicolas Bonnet \\ Centre d'études de l'emploi, Noisy-le-Grand
}

\begin{abstract}
A u regard de la mutation rapide des procédés d'échange Ainformationnels, les recherches actuelles en géographie économique tentent de saisir tant la nature sociale que les incidences de la proximité sur les échanges économiques et les territoires. L'évolution radicale contemporaine de sa définition participe à la modification des externalités qu'elle tend à produire. Ces externalités constituent un des facteurs clés de l'émergence de l'innovation ${ }^{1}$ qui ne se formalisent que dans une connectivité physique et relationnelle des agents innovateurs.

De cette organisation entrepreneuriale émerge une structuration sociale fondée sur des pratiques collaboratives et une capacité

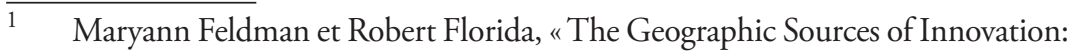
Technological Infrastructure and Product Innovation in the United States ", Annals of the Association of American Geographers, vol. 84, n 2, 1994, p. 210 229; Maryann Feldman et Nadine Massard, "Location, Location, Location: Institutions and Systems in the Geography of Innovation ", dans Maryann Feldman et Nadine Massard (dir.), Institutions and Systems in the Geography of Innovation, Norwell, Dordrecht, Kluwer Academic Publishers, 2002, p. 1-20.
\end{abstract}


$\mathrm{d}^{\prime}$ apprentissage mutuel ${ }^{2}$. Cependant, l'analyse des réseaux de partenariats de structures innovantes, notamment dans les biotechnologies ${ }^{3}$, montre que la survie d'entreprises de petite taille fréquemment présentes dans des districts reste fortement tributaire d'entreprises exogènes auxquelles elles restent liées, ne serait-ce que par des rapports financiers (financement direct, co-propriété de brevet). Au regard de l'évolution constante des liens entre les entreprises innovantes, de cet équilibre entre proximité géographique et organisationnelle, la position d'acteurs pivots dans les liens entre entreprises d'un même territoire peut conduire à l'évolution et au développement bénéfique de ce dernier. Ces acteurs pivots peuvent être présents sur le territoire de référence comme à mille autres lieux du globe. Leurs actions indirectes au sein du réseau d'acteurs, par leur position hiérarchique souvent ponctuelle au gré des dépôts de brevets, induisent le degré de résilience plus ou moins grand d'un territoire. Nous posons donc comme hypothèse de travail qu'une analyse globale d'un réseau local d'entreprises permet de déterminer le niveau de résilience d'un territoire. Cette étude sur plusieurs échelles de la morphologie d'un réseau offre la possibilité d'anticiper le degré d'adaptation d'un système productif et innovant local lors de perturbations économiques. Cette disposition résiliaire du réseau local influence indirectement le niveau de l'emploi sur une échelle de temps relativement courte.

Dans cet article, nous focalisons notre analyse sur le territoire d'une aire urbaine du sud de la France, Montpellier, qui jouit actuellement d'un développement important du nombre des petites et moyennes entreprises innovantes. Dans une première partie, nous exposerons la méthodologie de construction d'une

2 Mark Granovetter, "The strength of weak ties: A network theory revisited ", dans Peter Marsden et Nan Lin (dir.), Social Structure and Network Analysis, Beverly Hills, Sage Publications, 1982, p. 105-130.

3 Brigitte Gay et Bernard Dousset, "Innovation and network structural dynamics: Study of the alliance network of a major sector of the biotechnology industry ", Research Policy, n 34, 2005, p. 1457-1475.

4 Yannick Lung, Alain Rallet et André Torre, "Connaissances et proximité géographiques dans les processus d'innovation ", Géographie, Économie, Société, vol. 1, n², 1999, p. 281-306. 
base de données essentiellement composée de recensements de dépôts communs de brevets ${ }^{5}$. Dans une seconde partie, l'analyse morphologique des liens entre acteurs vise à apporter des éléments de compréhension dans le mécanisme résiliaire en l'associant tant à un territoire qu'à un environnement productif spécifique, en l'occurrence innovant. Dans une troisième partie, nous analyserons la corrélation qui se crée entre la morphologie d'un réseau de coopération et le niveau de l'emploi des clusters concernés.

\section{La résilience comme approche opératoire du potentiel partenarial innovant du territoire d'étude}

La résilience est intrinsèquement liée dans la littérature scientifique en économie géographique au district industriel. Elle postule tout d'abord, comme point de départ de cette faculté de retournement industriel, l'atomisation en petites entreprises de ces territoires productifs ${ }^{6}$. Alors que la fragmentation industrielle pourrait conduire à la vulnérabilité et à la faiblesse économique, au sein de la Silicon Valley elle apparaît comme une variable de flexibilité et d'ajustement pour Anna Lee Saxenian ${ }^{7}$. De cette organisation entrepreneuriale émerge une structuration sociale fondée sur des pratiques collaboratives et une capacité d'apprentissage mutuel. Cependant, l'analyse des réseaux de partenariats de structures innovantes, notamment dans les biotechnologies ${ }^{8}$,

$5 \quad$ Kelly Porter, Bunker Kjersten Whittington et Walter Powell, «The Institutional Embeddedness of High-Tech Regions: Relational Fondations of the Boston Biotechnology Community ", dans Stefano Breschi et Franco Malerba (dir.), Clusters, Networks, and Innovation, New York, Oxford University Press, 2005, p. 261-296; Adam Jaffe, Manuel Trajtenberg et Rebecca Henderson, "Geographic Localization of Knowledge Spillovers as Evidenced by Patent Citations ", dans Adam Jaffe et Manuel Trajtenberg (dir.), Patents, Citations, and Innovations: A Window on the Knowledge, Cambridge, MIT Press, 2002, p. 155-177.

6 Gabi Dei Ottati, "The remarkable resilience of the industrial districts of Tuscany ", dans Philip Cooke, Martin Heidenreich et Hans-Joachim Braczyk (dir.), Regional Innovation Systems: The role of governances in a globalized world, New York, London, Routledge, 2004, p. 21-43.

7 Anna Lee Saxenian, Regional advantages: Culture and competition in Silicon Valley and Route 128, Cambridge, Harvard University Press, 1994, 226 p.

8 Brigitte Gay et Bernard Dousset, op. cit. 
montre que la survie d'entreprises de petite taille fréquemment présentes dans des districts reste fortement tributaire d'entreprises exogènes auxquelles elles restent liées, ne serait-ce que par des rapports financiers (financement direct, co-propriété de brevet).

\subsection{Définition de la résilience des réseaux territoriaux}

Albert Réka et Albert-Laszlo Barabasi ${ }^{9}$ postulent que la robustesse d'un réseau est intrinsèquement liée à sa morphologie. Ainsi le niveau d'échelle de ses connectivités et la constitution de grands noyaux concentrant un nombre relativement dense de liens par rapport à des nœuds de moindre connectivité participent à la résilience des réseaux. Dans une approche en terme de systèmes complexes, c'est-à-dire où un grand nombre d'éléments interagissent de manière non prédictible les uns par rapport aux autres $^{10}$, la résilience correspond à l'intensité maximale de la force qu'un système peut absorber sans modifier son comportement et ses fonctions de régulation. Il s'agit pour un système de garantir le rétablissement de l'équilibre nécessaire au maintien de son intégrité. La résilience prend, dès lors, consistance au cours de fortes perturbations économiques endogènes ou exogènes affectant un territoire ${ }^{11}$. C'est donc cette capacité d'anticipation des risques et son incidence dans l'espace urbain que nous allons ici analyser. Il s'agit alors de comprendre le territoire productif moins comme une suite de localisations mais comme un système dynamique ou " système productif local " ${ }^{12}$ dans lequel les acteurs

$9 \quad$ Albert Réka et Albert-Laszlo Barabasi, "Statistical mechanics of complex networks ", Reviews of Modern Physics, n 74, 2002, p. 47-97.

10 Stanly Holling Crawford, "Resilience and Stability of Ecological Systems ", Annual Review of Ecology and Systematics, vol. 4, 1973, p. 1-23; Lance Gunderson, "Ecological Resilience - In Theory and Application », Annual Review of Ecology and Systematics, vol. 31, 2000, p. 425-439.

11 Christina Aschan-Leygonie, "Vers une analyse de la résilience des systèmes spatiaux ", L'Espace géographique, n 1, 2000, p. 64-77; Gabi Dei Ottati, "The remarkable resilience of the industrial districts of Tuscany ", op. cit. Claude Courlet, "Les systèmes productifs localisés, de quoi parle-t-on? ", dans Claude Courlet et Soulage Bernard (dir.), Industrie territoires et politiques publiques, Paris, L'Harmattan, 1994, p. 22. 
interagissent sur plusieurs niveaux scalaires ${ }^{13}$ et orientent en circonstance leurs actions.

1.2. Adaptation de la résilience au système d'emploi des réseaux d'innovation

La mise en réseaux d'entreprises innovantes stimule l'innovation de chaque entreprise et stimule donc par ailleurs l'activité propre de chacune d'elles. En cela, l'innovation peut être considérée comme un facteur local d'emploi qui participe à discriminer géographiquement les territoires les uns par rapport aux autres ${ }^{14}$. Létude de l'évolution des brevets sur un territoire déterminé est donc un facteur indicatif dans la définition de la résilience d'un territoire au cours de périodes de moindre activité économique, assimilables ainsi à des perturbations révélatrices du phénomène.

La théorie des graphes constitue une méthode d'analyse désormais commune pour les géographes et aménageurs s'intéressant aux réseaux d'infrastructures de transport mais également aux réseaux de communications électroniques. Elle permet, en outre, une analyse objective des relations de coopérations productives entre entreprises de secteurs et de territoires différents. Cette réalité entrepreneuriale trouve une concrétisation productive et géographique mais aussi, à juste titre, structurante pour les territoires, au même titre que peuvent l'être des réseaux techniques. La structure de graphes de relations entre entreprises et la lecture de ses propriétés par la théorie des graphes renseignent significativement sur la genèse des combinaisons réticulaires d'entreprises. Contrairement aux districts industriels, les clusters reposent sur une double composante à la fois territorialisée et aspatiale qui rend difficile leur étude voire même leur repérage. Les externalités de réseaux constituent une des principales caractéristiques du développement des clusters. Ces derniè-

13 Frank Auriac, "Région-Système : Région et Systèmes économiques ", L'Espace géographique, $\mathrm{n}^{\circ}$ 4, 1986, p. 272-277.

14 Rodolphe Dodier, "La dimension spatiale du marché de l'emploi », Hommes et terres du Nord, $\mathrm{n}^{\circ} 1,2001$, p. 3-9. 
res apparaissent dès lors que « la satisfaction que retire un agent de son adhésion à un réseau économique est positivement corrélée au nombre d'adopteurs de ce réseau $" 15$.

\subsection{Données}

Le protocole relatif à la constitution de la base sur les dépôts communs de brevets se définit comme suit. Nous avons utilisé le registre des dépôts de brevets auprès de l'Organisation mondiale de la propriété intellectuelle (OMPI). Par la recherche d'harmonisations entre les différentes normes et procédures des états relatives à la propriété intellectuelle, cet organisme des Nations Unies fournit, d'une part, une classification internationale des brevets (CIB) et constitue, d'autre part, une base commune à la propriété intellectuelle des inventions. Cette base de données répertorie les demandes internationales de brevet autrement appelé PCT (Patent Cooperation Treaty). Le système PCT ne constitue ni un brevet international ni la garantie d'une acceptation globale de la demande de brevet par l'ensemble des organismes nationaux ou régionaux de propriété intellectuelle qui demeurent seuls juges dans la recevabilité de la demande. Cependant, il permet une protection de l'invention durant un temps limité. L'utilisation de cette base offre un plus large spectre de la propriété intellectuelle au niveau mondial sans pour autant constituer un titre de propriété industrielle. Nous avons donc bâti ici une base de liens de coopérations entre entreprises selon les dépôts communs de brevets auprès de cet organisme dans les domaines des biotechnologies et des TIC et selon la classification de l'OMPI (voir Annexe). La base a été construite à partir des adresses des inventeurs et non de celles des déposants afin d'obtenir une localisation précise des agents innovateurs. En effet, les déposants étant fréquemment les maisons-mères des firmes, aucune interprétation spatiale de la territorialisation de l'innovation n'aurait pu être réalisée.

15 Jérôme Vicente, "Externalités de réseaux versus externalités informationnelles dans les dynamiques de localisation ", Revue d'économie régionale et urbaine, $\mathrm{n}^{\circ} 4,2002$, p. 5. 


\subsection{Méthodologie}

La constitution de graphes de données d'entreprises permet d'analyser des réseaux de coopération au sein d'un cluster. Une telle analyse offre la possibilité de suivre le cheminement de ces coopérations dans un réseau selon la nature et le besoin des compétences mises en œuvre. En effet, dans le cadre d'un projet de développement, certaines entreprises s'appuient sur des entreprises relais au sein d'un réseau afin d'en atteindre d'autres plus aisément et donc d'obtenir l'information technologique garante de la compétitivité du produit final. La circulation continuelle de l'information par des canaux tout autant formels qu' informels est censée assurer la pérennité de l'activité innovante. Au sein de ces réseaux auto-organisés à l'image des clusters, les comportements rationnels et opportunistes des entreprises sont guidés par l'accélération du temps créatif et productif imposé par le marché globalisé. Pour formaliser les graphes de relations, Brigitte Gay et Bernard Dousset ${ }^{16}$ proposent une modélisation informatique des graphes d'entreprises ainsi qu'une interprétation fondée notamment sur l'émergence des centralités et la formation des cliques qui y sont liées.

La technique de visualisation du graphe utilisée ici s'appuie sur l'algorithme de Fruchterman d'attraction entre sommets liés puis de répulsion à l'endroit de l'ensemble des sommets du graphe. L'analyse de l'évolution de la structure du réseau de coopération par les liens formels que constituent les dépôts de brevets offre une double analyse. Tout d'abord, elle permet de situer au sein d'un réseau les organismes relais, institutions ou entreprises qui constituent les centralités et donc les charnières de ces réseaux. Dans un second temps, l'étude morphologique évolutive positionne spatialement les relais de ces réseaux et détermine un certain niveau d'efficience territoriale. La combinaison de ces deux ensembles entrepreneuriaux et territoriaux détermine conjointement le système innovateur et productif de l'aire urbaine montpelliéraine.

$\overline{16}$ Brigitte Gay et Bernard Dousset, op. cit. 


\section{Une analyse de la résilience d'un réseau local d'entreprises innovantes}

Selon la théorie des graphes, les caractéristiques des réseaux diffèrent ici dans leur globalité selon deux indicateurs principaux.

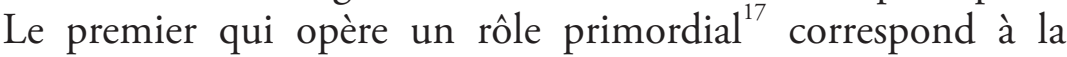
connexité. Il s'exprime notamment par le rapport entre le nombre de liens observés dans un réseau et le nombre de nœuds ${ }^{18}$. Lorsque cette connexité est supérieure à 1 , cela signifie que le graphe gagne en complexité. Ainsi pour un nombre donné de nœuds, plus il y a de liens, plus grand est le choix de relation d'un nœud à l'autre. Le graphe de l'ensemble de la période d'étude 1997-2006 présente une forte connexité avec un indice de 1,48. Également, entre la période 1997-2003 $(1,18)$ et la période 2003-2006 (1,72), le réseau a fortement gagné en connexité.

\section{Tableau 1}

Statistiques descriptives de l'analyse des brevets

\begin{tabular}{|l|l|l|l|l|l|}
\hline Période & $\begin{array}{l}\text { Part de liens } \\
\text { issus de } \\
\text { brevets } \\
\text { publics-privés } \\
(\text { en \%) }\end{array}$ & $\begin{array}{l}\text { Part de liens } \\
\text { issus de brevets } \\
\text { entre } \\
\text { établissements } \\
\text { privés (en \%) }\end{array}$ & $\begin{array}{l}\text { Nombre de } \\
\text { liens entre } \\
\text { établissements } \\
\text { issus de } \\
\text { brevets } \\
\text { communs }\end{array}$ & $\begin{array}{l}\text { Centralité } \\
\text { d'intermédiarité } \\
\text { moyenne entre } \\
\text { nœuds }\left(^{*}\right)\end{array}$ & $\begin{array}{l}\text { Part } \\
\text { d'entreprises } \\
\text { récurrentes } \\
\left(\text { en \%) }\left(^{* *}\right)\right.\end{array}$ \\
\hline $1997-2003$ & 3,5 & 36 & 86 & 0,37 & 36 \\
\hline $2003-2006$ & 2,3 & 33 & 175 & 0,56 & 67 \\
\hline $1997-2006$ & 2,7 & 34 & 261 & 0,53 & 57 \\
\hline
\end{tabular}

(*) Également dénommé Average betweenness centrality

(**) Entreprises participant à au moins deux dépôts de brevets communs durant la période concernée.

\section{Source : OMPI, traitement de l'auteur, N.Bonnet, 2009}

17 Gabriel Dupuy, «Géographie et économie des réseaux », L’Espace géographique, $\mathrm{n}^{\circ} 3,1993$, p. 193-209.

18 La connexité se définie comme " une propriété essentielle des réseaux, qui caractérise leur capacité à mettre en relation tous les nœuds du territoire qu'ils desservent. Ainsi un graphe est dit connexe si, quels que soient deux sommets distincts, il existe une chaine entre deux sommets " (Denise Pumain et Thérèse Saint-Julien, L'analyse spatiale. Localisation dans l'espace, Paris, Armand Colin, 1997, p. 96). L'indicateur de cette connexité que nous choisissons d'utiliser ici est l'indice $\beta=L / N$. 
Le second indicateur est celui d'average betweenness centrality du réseau ou centralité d'intermédiarité moyenne (tableau 1). Il s'agit de la fréquence à laquelle un noud se trouve sur le chemin le plus court entre des paires de nouds. Elle s'applique pour apprécier la situation centrale de chacun des sommets du graphe. Dans le cas présent, elle correspond à une moyenne pour l'ensemble du graphe. Cependant dans l'absolu, une entreprise peut avoir une position au sein du réseau, soit centrale si elle est largement connectée aux autres nœuds du réseau, soit périphérique si cette connectivité est faible. Nous n'effectuons pas ici de mesure statistique de la centralité de chacun des nœuds, nous nous cantonnant à leur interprétation depuis le graphe. Néanmoins, la centralité et son calcul au moyen de la centralité de degré, en permettant de caractériser la position dominante d'un noeud au sein du réseau, confère à l'agent central un certain pouvoir initiateur dans la dynamique évolutive du réseau.

Figure 1

Coopérations formelles d'innovation entre entreprises sur la période 1997 à 2006

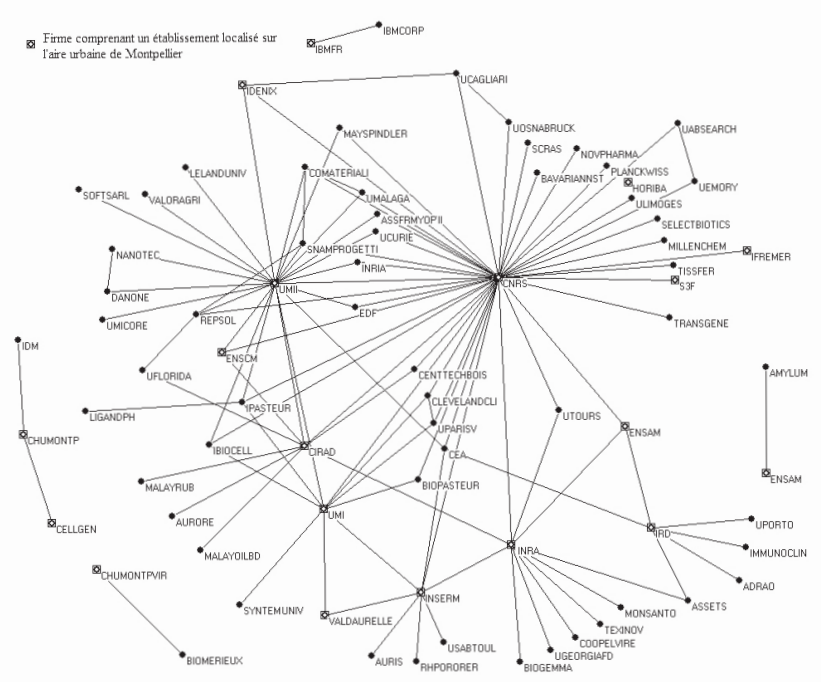

Source : OMPI, N. Bonnet, 2006 
Le graphe décrivant l'ensemble des coopérations (période $1997-$ 2006) et représenté par la figure 1 donne une visualisation globale du réseau d'innovation montpelliérain. Il permet, par comparaison avec les deux autres périodes (figures 2 et 3), de constater que, outre des acteurs stratégiques essentiellement institutionnels, peu d'acteurs présentent la capacité de figurer sur les deux phases de l'étude. Ces deux phases semblent correspondre à deux vagues technologiques distinctes sur le territoire montpelliérain tout en coïncidant avec une densification du réseau dans les secteurs innovants. Une entreprise innovante, pour le rester, doit soit densifier son réseau soit atteindre une taille critique.

Figure 2

Coopérations formelles d'innovation entre entreprises sur la période 1997 à 2003

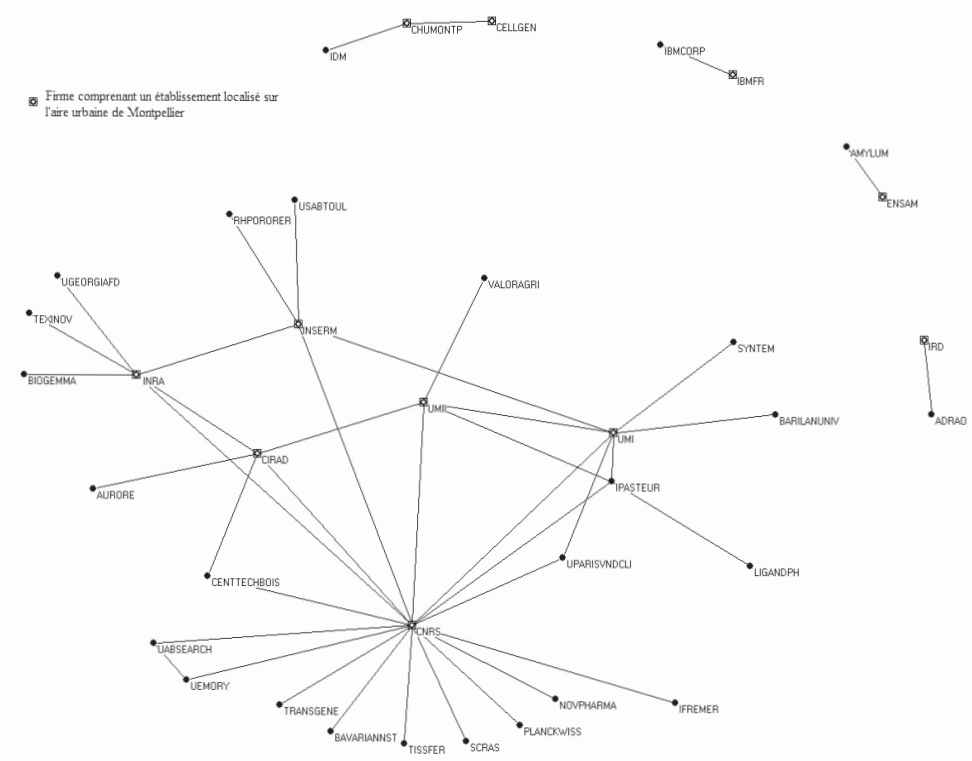

Source : OMPI, N. Bonnet, 2006

Les acteurs centraux (ceux qui concentrent autour d'eux le plus de coopérations), essentiellement les centres de recherche institutionnels issus principalement du CNRS et de l'Université Montpellier-II, détiennent de fait, à titre collectif, la grande 
majorité des brevets dans le domaine des biotechnologies et des TIC sur l'ensemble de la période traitée (figure 1). Le cœur de compétence de ces acteurs participe donc à structurer le réseau. Ainsi paradoxalement, durant la période 1997-2003 (figure 2) dominée par un acteur principal, le CNRS, le réseau semble être tout à la fois contracté autour de cette institution majeure, le diamètre et le chemin moyen entre deux acteurs sont extrêmement courts mais la connexité générale du réseau est nettement supérieure à ce qu'elle sera dans la période suivante, pour preuve le nombre de cliques indépendantes et périphériques au noyau central. A contrario, durant la période suivante 2003-2006 (figure 3), le réseau présente certes deux centralités majeures autour du CNRS et de l'Université Montpellier-II mais semble se relâcher en de multiples et petites centralités périphériques consacrant une certaine diffusion de compétences.

Figure 3

Coopérations formelles d'innovation entre entreprises sur la période 2003 à 2006

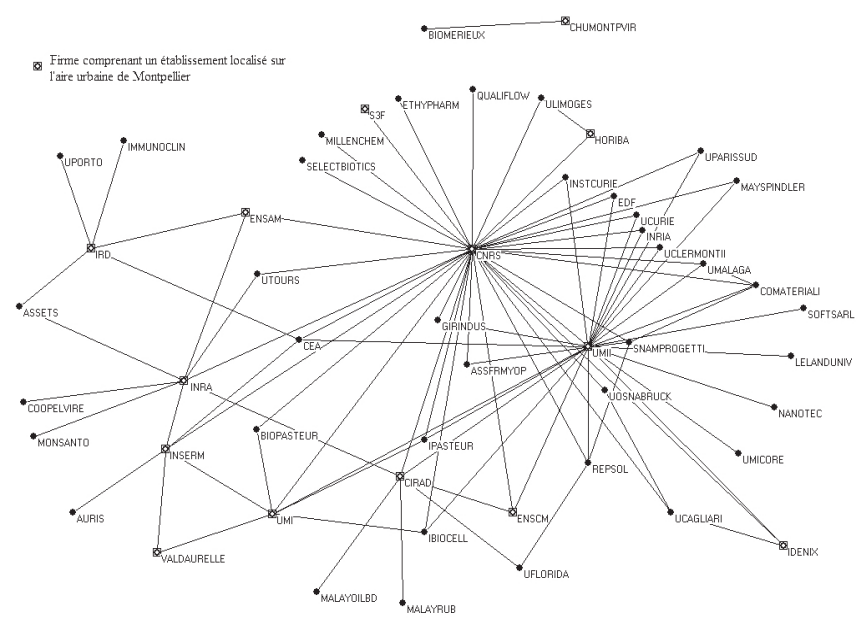

Source : OMPI, N. Bonnet, 2006 
Le comparatif entre les deux périodes de référence apporte des renseignements sur la capacité évolutive du réseau. Si les nœuds centraux ont perduré entre les deux dates, ce qui apparaît marquant, c'est la régénérescence de ce réseau qui se caractérise par sa plus grande connexité sur la période 2003-2006 et sa capacité à avoir intégré de nouveaux entrants, essentiellement des entreprises privées et hors du territoire montpelliérain. Cependant, cela signifie que le nombre tout aussi important d'entreprises montpelliéraines à avoir déposé des brevets l'a fait en collaboration avec des entreprises exogènes accroissant ainsi la connectivité extérieure du territoire et donc l'étendue géographique du réseau. La plus grande imbrication d'échelles qui s'affirme, confirme également le système territorial montpelliérain comme un milieu innovateur, productif dans le présent mais garantissant une pérennité dans les productions futures.

Par ailleurs, la densification liée à une certaine diversification des nouveaux entrants se caractérise par la formation de cliques périphériques par rapport à la centralité toujours renforcée des institutions de recherche publique, en premier lieu, le CNRS. Alors que ce dernier fédère des universités étrangères essentiellement européennes et nord-américaines, le second centre principal qui émerge sur la période 2003-2006 est l'Université des Sciences. La globalité que celle-ci représente est constituée des différents laboratoires affiliés, et ses coopérations sont dirigées à parts égales avec d'autres institutions et entreprises.

\section{Lien de coopération et incidence en termes d'emploi local}

Sur les deux périodes de référence, Montpellier s'est affirmée comme un territoire innovant. Ses liens se sont diversifiés dans le temps, à la fois au niveau des échelles spatiales mais aussi entre secteurs d'activité. L'ensemble de ces paramètres tend à augmenter la potentialité de résilience du système territorial montpelliérain. C'est ainsi que la résilience systémique d'un territoire productif correspond au résultat combinatoire d'un milieu interne, du territoire lui-même et de son environnement exogène. 
En effet, le contexte plus vaste auquel est lié le système territorial, c'est-à-dire les liens transnationaux, l'assise politique et socioculturelle nationale et dont il tire les ressources matérielles, financières ou symboliques nécessaires à son fonctionnement et à sa survie, peut également produire des modifications majeures ${ }^{19}$. Cette symbiose économique et spatiale confirme que la capacité résiliaire d'un territoire résulte de l'aptitude ubiquiste de son système productif à tisser des liens indépendamment de toute considération spatiale, seulement mue par les besoins d'acquisition de compétences spécifiques. Ainsi, proximité et connectivité des entreprises ne présentent guère de corrélation, tout comme l'usage des TIC ne contribuent guère plus à l'interaction entre agents innovateurs, car « la raison la plus évidente qui pousse la firme à adhérer au réseau réside en fait dans la possibilité de contacter plus facilement et plus rapidement certains groupes de firmes..." "

Dans le cas de Montpellier, le secteur des biotechnologies, bien que plus récent que celui des TIC, présente un niveau de connectivité local bien plus important. L'environnement historique lié au secteur hospitalier et médical explique en partie les fortes relations entre la recherche et la création d'entreprises par suite de dépôts de brevets. Ces liens étroits entre recherche publique, privée et entreprises commerciales se révèlent pérennes ou en constant renouvellement dans la vie d'une entreprise biotechnologique innovante. En effet, la rapide obsolescence des connaissances dans les domaines des biotechnologies nécessite un renouvellement constant de leurs bases scientifiques. Ce particularisme dû au haut degré d'innovation du secteur peut induire, de ce fait, un différentiel de niveau de survie des petites entreprises innovantes entre les deux secteurs de référence. Durant la période d'étude, la démographie d'entreprise et, par

19 Alexandre Steyer et Jean-Benoît Zimmermann, «Influence sociale et diffusion de l'innovation ", Mathematics and Social Sciences, $n^{\circ}$ 168, 2004, p. 43-57.

20 Roberta Capello et Peter Nijkamp, "Le rôle des externalités de réseau dans les performances des firmes et des régions : l'exemple des NTIC ", dans Alain Rallet et André Torre (dir.), Économie industrielle et économie spatiale, Paris, Economica, 1995, p. 285. 
voie de conséquence, le chômage présentent également des fluctuations corrélées avec le niveau de résilience du réseau. Néanmoins, la situation paradoxale de l'emploi en LanguedocRoussillon ne permet pas dans un premier temps une corrélation directe avec le niveau de retour à l'emploi. En effet, cette région conjugue certes un taux de chômage parmi les plus forts de la France métropolitaine, mais également un afflux démographique conséquent et un important taux de création d'entreprises.

À titre de comparaison, mais en usant d'un autre indicateur puisque nos données ne portent que sur l'étude de l'aire urbaine de Montpellier, le système productif de cette dernière présente un singulier paradoxe au regard des données nationales depuis une dizaine d'années. En effet, la région de Montpellier conjugue à la fois un taux de création d'entreprises, supérieur au niveau national (14\% contre $12 \%$ ) et un taux de survie à cinq ans inférieur au taux national (50\% contre $56 \%$ ). L'écrasante majorité de ces créations concerne des entreprises sans salarié. Lautre élément statistique marquant est la prépondérance de la recherche publique sur la recherche privée même si, comme le montre notre étude, les partenariats publics-privés tendent à s'accroître $^{21}$. Par ailleurs, une des particularités de Montpellier par rapport aux autres métropoles françaises est l'extrême atomisation de son système productif et innovant essentiellement composé de PME. Cette situation structurelle de l'industrie locale constitue notamment un des éléments de la sélection de Montpellier au sein de la région Languedoc-Roussillon comme zone pilote de l'Union Européenne pour un suivi des fonds JEREMIE (Joint European Resources for Micro-to-Medium Enterprises). Ce fond européen en direction des petites et moyennes entreprises est géré par le Fond Européen d'Investissement dans le cadre des programmes européens courant de 2007 à 2013.

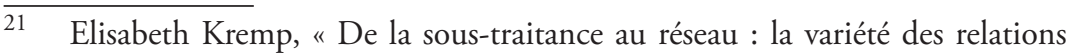
inter-entreprises », INSEE Méthodes, nº 109, 2005, 4 p. 


\section{Conclusion}

L'analyse de ces réseaux sur différentes échelles géographiques permet ainsi de caractériser l'évolution résiliaire d'un territoire. Cette caractéristique résiliaire du territoire apparaît au travers de l'étude de la démographie des entreprises en particulier innovantes durant la période de référence. Cependant, la mise en évidence de cette résilience n'apparaît concrètement que lors de perturbations. Dans le graphe, les propriétés résiliaires d'un réseau se traduisent dans sa morphologie globale. En effet, c'est la connexité du réseau de liens de coopération ainsi que la perduration de ces liens qui assurent la capacité de recouvrement d'un territoire. Cette analyse nous aura permis d'identifier un certains nombre d'acteurs-pivots qui garantissent la viabilité sur le long terme du territoire d'étude. Néanmoins, la démographie d'entreprises prend une certaine part dans l'explication du phénomène de résilience en raison du caractère stratégique que représente l'innovation dans le dynamisme prouvé ou supposé d'un territoire. La coopération entre des entreprises crée un volume d'échange et d'activité sur un territoire qui est fonction de leur taille et de leur ancienneté. Une piste de recherche suivante serait d'approfondir l'analyse de la structure des réseaux d'entreprises en vue de la confronter avec un panel de variables susceptibles de prédéterminer la coopération des entreprises innovantes sur un territoire.

\section{Bibliographie}

Aschan-Leygonie, Christina, "Vers une analyse de la résilience des systèmes spatiaux ", L'Espace géographique, n 1, 2000, p. 64-77.

Auriac, Frank, "Région-Système : Région et Systèmes économiques ", L'Espace géographique, n 4, 1986, p. 272-277.

Autant-Bernard, Corinne, Jacques Mairesse et Nadine Massard, "Spatial knowledge diffusion through collaborative networks ", Papers in Regional Science, vol. 86, n 3, 2007, p. 341-350. 
Réka, Albert et Albert-Laszlo Barabasi, «Statistical mechanics of complex networks ", Reviews of Modern Physics, $\mathrm{n}^{\circ}$ 74, 2002, p. 47-97.

Capello, Roberta et Peter Nijkamp, «Le rôle des externalités de réseau dans les performances des firmes et des régions : l'exemple des NTIC », dans Alain Rallet et André Torre (dir.), Économie industrielle et économie spatiale, Paris, Economica, 1995, p. 273-293.

Courlet, Claude, "Les systèmes productifs localisés, de quoi parle-t-on? ", dans Claude Courlet et Soulage Bernard (dir.), Industrie territoires et politiques publiques, Paris, L'Harmattan, 1994, p. 13-32.

Dodier, Rodolphe, "La dimension spatiale du marché de l'emploi ", Hommes et terres $d u$ Nord, $\mathrm{n}^{\circ}$ 1, 2001, p. 3-9.

Dupuy, Gabriel, "Géographie et économie des réseaux ", L'Espace géographique, $\mathrm{n}^{\circ} 3,1993$, p. 193-209.

Feldman, Maryann et Robert Florida, «The Geographic Sources of Innovation: Technological Infrastructure and Product Innovation in the United States", Annals of the Association of American Geographers, vol. 84, n 2, 1994, p. 210-229.

Feldman, Maryann et Nadine Massard, "Location, Location, Location: Institutions and Systems in the Geography of Innovation ", dans Maryann Feldman et Nadine Massard (dir.), Institutions and Systems in the Geography of Innovation, Norwell, Dordrecht, Kluwer Academic Publishers, 2002, p. 1- 20.

Gay, Brigitte et Bernard Dousset, "Innovation and network structural dynamics: Study of the alliance network of a major sector of the biotechnology industry ", Research Policy, n 34, 2005, p. 14571475 .

Granovetter, Mark, "The strength of weak ties: A network theory revisited ", dans Peter Marsden et Nan Lin (dir.), Social Structure and Network Analysis, Beverly Hills, Sage Publications, 1982, p. 105130.

Gunderson, Lance, «Ecological Resilience - In Theory and Application », Annual Review of Ecology and Systematics, vol. 31, 2000, p. 425-439.

Holling Crawford, Stanley, "Resilience and Stability of Ecological Systems ", Annual Review of Ecology and Systematics, vol. 4, 1973, p. 1-23.

Jaffe, Adam, Manuel Trajtenberg et Rebecca Henderson, " Geographic Localization of Knowledge Spillovers as Evidenced by Patent Citations ", dans Adam Jaffe et Manuel Trajtenberg (dir.), Patents, Citations, and Innovations: A Window on the Knowledge, Cambridge, MIT Press, 2002, p. 155-177. 
Kremp, Elisabeth, « De la sous-traitance au réseau : la variété des relations inter-entreprises ", INSEE Méthodes, n 109, 2005, 4 p.

Lung, Yannick, Alain Rallet et André Torre, "Connaissances et proximité géographiques dans les processus d'innovation ", Géographie, Économie, Société, vol. 1, n² 2, 1999, p. 281-306.

Ottati, Gabi Dei, "The remarkable resilience of the industrial districts of Tuscany ", dans Philip Cooke, Martin Heidenreich et Hans-Joachim Braczyk (dir.), Regional Innovation Systems: The role of governances in a globalized world, New York, London, Routledge, 2004, p. 21-43.

Porter, Kelley, Kjersten Bunker Whittington et Walter Powell, "The Institutional Embeddedness of High-Tech Regions: Relational Fondations of the Boston Biotechnology Community ", dans Stefano Breschi et Franco Malerba (dir.), Clusters, Networks, and Innovation, New York, Oxford University Press, 2005, p. 261-296.

Pumain, Denise et Thérèse Saint-Julien, L'analyse spatiale. Localisation dans l'espace, Paris, Armand Colin, coll. "Cursus géographie ", 1997, $161 \mathrm{p}$.

Saxenian, Anna Lee, Regional advantages: Culture and competition in Silicon Valley and Route 128, Cambridge, Harvard University Press, 1994, $226 \mathrm{p}$.

Saxenian, Anna Lee et Jinn-Yuh Hsu, "The Silicon Valley-Hsinchu Connection: Technical Communities and Industrial Upgrading ", dans Stefano Breschi et Franco Malerba (dir.), Clusters, Networks, and Innovation, New York, Oxford University Press, 2005, p. 235-260.

Steyer, Alexandre et Jean-Benoît Zimmermann, "Influence sociale et diffusion de l'innovation ", Mathematics and Social Sciences, n 168, 2004, p. 43-57.

Veltz, Pierre, Mondialisation, Villes et territoires. L'économie d'archipel, Paris, PUF, coll. "Économie en liberté », [1996] 2000.

Vicente, Jérôme, «Externalités de réseaux versus externalités informationnelles dans les dynamiques de localisation ", Revue d'économie régionale et urbaine, $\mathrm{n}^{\circ}$ 4, 2002, p. 535-552. 


\section{Annexe}

Note méthodologique et descriptive sur l'analyse des brevets

Une liste des coopérations a été élaborée à la date des dépôts de brevets sur la période du 22/09/1997 au 17/02/2006 à partir de cette base de données de l'OMPI. Pour réaliser l'analyse, nous avons procédé à un découpage temporel de notre base de données des dépôts communs de brevets en deux grandes périodes de 1997 à 2003 et de 2003 à 2006. Au regard du faible nombre de dépôts de brevets PCT durant la première partie des dix années sur laquelle porte l'étude, ce découpage se justifie notamment par la nécessité de faire primordialement apparaître les entreprises pivots. Cet impératif impliquait donc de recueillir un nombre suffisant de dépôts communs de brevets sur une échelle de temps assez longue pour repérer ces entreprises innovantes multipartenaires. Par ailleurs, l'essentiel du potentiel innovant du système territorial montpelliérain repose sur les deux secteurs de référence de notre étude. En cela, mis à part certains aspects spécifiques de l'activité médicale très présente également sur Montpellier, les brevets issus de la recherche médicale ont été incorporés dans notre base en raison de la proximité disciplinaire des équipes et de l'intrication des réseaux de coopération. À ce titre, la base de données ainsi produite se caractérise par l'exhaustivité du référencement des réseaux d'innovation présents sur l'aire urbaine de Montpellier. Cependant, cette base ne peut être considérée que comme un complément de données relatives aux coopérations entre établissements compte tenu que, d'une part, toutes les coopérations ne portent pas nécessairement sur une thématique innovante, mais que, d'autre part, celles en recherche et développement ne débouchent pas systématiquement sur le dépôt de brevets. Par ailleurs, ce dépôt peut être interprété de deux manières radicalement différentes, soit comme l'aboutissement réussi de coopérations innovantes régulières, en cela des effets de débordement peuvent entrer en considération, soit comme le résultat de recherches ayant donné lieu à des collaborations ins- 
titutionnelles purement formelles et essentiellement financières. Une autre limite peut être posée à l'utilisation de cette base et qui constitue une constante dans les grandes bases statistiques, c'est la présence de boîtes noires sous la forme des grandes institution comme le CNRS ou les universités, et qui ne permet donc pas de désigner clairement quel laboratoire se trouve effectivement à l'origine de la recherche. Néanmoins, dans ce cas de figure, nous nous sommes basés sur l'adresse des inventeurs. Dès lors que leur adresse était majoritairement montpelliéraine nous avons fait le choix de comptabiliser le CNRS comme laboratoire local. Par ailleurs, cela confirme le rôle toujours majeur de la recherche publique mais surtout démontre également le lien croissant entre cette dernière et la recherche privée. Durant la période retenue, 673 dépôts de brevets ont concerné au moins un chercheur domicilié sur Montpellier. Quatre-vingt-douze (92) entreprises ont déposé des brevets sur la base minimum d'un binôme, permettant ainsi de définir des liens de coopération. 